\begin{tabular}{|c|c|c|c|c|c|c|}
\hline \multirow{4}{*}{ Impact Factor: } & ISRA (India) & $=3.117$ & SIS (USA) & $=0.912$ & ICV (Poland) & $=6.630$ \\
\hline & ISI (Dubai, UAE & $=0.829$ & РИНЦ (Russia) & $=0.156$ & PIF (India) & $=1.940$ \\
\hline & GIF (Australia) & $=0.564$ & ESJI (KZ) & $=8.716$ & IBI (India) & $=4.260$ \\
\hline & JIF & $=1.500$ & SJIF (Morocco) & $=5.667$ & OAJI (USA) & $=0.350$ \\
\hline
\end{tabular}

\begin{tabular}{|c|c|}
\hline $\begin{array}{l}\text { SOI: } \underline{1.1 / \mathrm{TA}} \\
\text { International Sc } \\
\text { Theoretical } \mathbf{\&}\end{array}$ & $\begin{array}{l}\underline{S} \text { DOI: } 10.15863 / T A S \\
\text { ientific Journal } \\
\text { pnlied Science }\end{array}$ \\
\hline p-ISSN: 2308-4944 (print) & e-ISSN: $2409-0085$ (online) \\
\hline Year: 2019 & Volume: 73 \\
\hline Published: 15.05.2019 & http://T-Science.org \\
\hline
\end{tabular}

SECTION 2. Applied mathematics.

Mathematical modeling.
QR - Issue
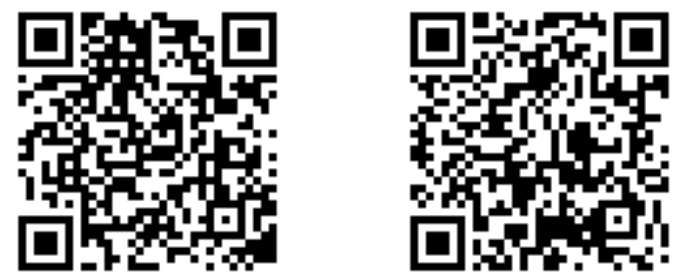

Umarali Abduvaxabovich Nazarov

Candidate of Physical and Mathematical Sciences,

Docent to department of information technology,

Samarkand state architectural and civil engineering institute, Uzbekistan umaralin@rambler.ru

\title{
INFLUENCE ON THE DISTRIBUTION DEFINES THE PARAMETERS OF DETONATION WAVES IN GAS SUSPENSION IN SHARP EXTENDABLE TUBULAR
}

Abstract: The model equations of two-dimensional axisymmetric unsteady motion of the reacting mixture of gas and particles monopropellant mathematical simulation of wave propagation mechanisms of combustion and detonation in heterogeneous dramatically expanding tubes. To study the influence of geometrical parameters of the pipe and the size of the particles in the spread of detonation waves in gas suspensions monopropelland.

Key words: burning, detonation, shock wave, mathematical model, monopropelland, the weight content of the particles.

Language: Russian

Citation: Nazarov, U. A. (2019). Influence on the distribution defines the parameters of detonation waves in gas suspension in sharp extendable tubular. ISJ Theoretical \& Applied Science, 05 (73), 40-46.

Soi: http://s-o-i.org/1.1/TAS-05-73-9 Doi: crossef https://dx.doi.org/10.15863/TAS.2019.05.73.9

\section{ВЛИЯНИЕ ОПРЕДЕЛЯЮЩИХ ПАРАМЕТРОВ НА РАСПРОСТРАНЕНИЕ ДЕТОНАЦИОННЫХ ВОЛН В ГАЗОВЗВЕСЯХ В РЕЗКО РАСШИРЯЮЩИХСЯ ТРУБАХ}

Аннотация: B рамках модели двухмерного осисимметричного нестационарного движения реагирующей смеси газа и частич унитарного топлива выполнено математическое моделирование механизмов распространения волн горения и гетерогенной детонации в резко расширяющихся трубах. Изучено влияния геометрических параметров трубопровода и размера частич на распространение детонационных волн в газовзвесях унитарного топлива.

Ключевые слова: горение, детонаџия, ударная волна, математическая модель, унитарного топлива, массовое содержание частии.

\section{Введение}

Проблема распространения детонационных волн в резко расширяющихся трубах была изучено численно и экспериментально в работах [3-9]. В настоящей работе, численно исследуется влияние основных определяющих параметров газовзвесей и трубопровода на процесс распространения нестационарных детонационных волн в резко расширяющихся трубах.

\section{Основные уравнения.}

Пусть имеется смесь газа с твердыми частицами унитарного топлива. Для описания ее движения используются следующие допущения $[1 ; 2]$ : расстояния, на которых параметры течения меняются существенно, много больше характерных размеров частиц и расстояний между ними; эффекты вязкости и теплопроводности существенны лишь в процессах взаимодействия газа с частицами; частицы унитарного топлива несжимаемы и имеют сферическую форму; смесь - локально монодисперсная; объемное содержание частиц аэровзвеси весьма незначительно $(0,1 \%)$, так, что столкновение между частицами не учитываются. Дополнительно предполагается, что процессы воспламенения и горения осуществляются при температуре частиц унитарного топлива, равной температуре разложения, а продукты горения взвеси, образующиеся в зоне химической 


\begin{tabular}{|c|c|c|c|c|c|c|}
\hline \multirow{4}{*}{ Impact Factor: } & ISRA (India) & $=3.117$ & SIS (USA) & $=0.912$ & ICV (Poland) & $=6.630$ \\
\hline & ISI (Dubai, UAE & $=0.829$ & РИНЦ (Russia) & $=0.156$ & PIF (India) & $=1.940$ \\
\hline & GIF (Australia) & $=0.564$ & ESJI (KZ) & $=8.716$ & IBI (India) & $=4.260$ \\
\hline & JIF & $=1.500$ & SJIF (Morocco) & $=5.667$ & OAJI (USA) & $=0.350$ \\
\hline
\end{tabular}

реакции, являются идеальным калорически совершенным газом.

В рамках принятых допущений система дифференциальных уравнений двухмерного осесимметричного нестационарного движения реагирующей смеси газа и частиц унитарного топлива может быть записана в следующем виде $[1 ; 2 ; 9]$ :

$$
\begin{aligned}
& \frac{\partial \rho_{1 k}}{\partial t}+\frac{\partial \rho_{1 k} v_{1 r} r}{r \partial r}+\frac{\partial \rho_{1 k} v_{1 z}}{\partial z}=(k-1) J \quad(k=1,2), \quad \frac{\partial \rho_{2}}{\partial t}+\frac{\partial \rho_{2} v_{2 r} r}{r \partial r}+\frac{\partial \rho_{2} v_{2 z}}{\partial z}=-J, \\
& \frac{\partial n}{\partial t}+\frac{\partial n v_{2 r} r}{r \partial r}+\frac{\partial n v_{2 z}}{\partial z}=0, \quad \alpha_{2}=\frac{1}{6} \pi d^{3} n \\
& \frac{\partial \rho_{i} v_{i r}}{\partial t}+\frac{\partial \rho_{i} v_{i r} v_{i r} r}{r \partial r}+\frac{\partial \rho_{i} v_{i r} v_{i z}}{\partial z}+(2-i) \frac{\partial p}{\partial r}=(-1)^{i}\left(F_{r}-J v_{2 r}\right) \\
& \frac{\partial \rho_{i} v_{i z}}{\partial t}+\frac{\partial \rho_{i} v_{i r} v_{i z} r}{r \partial r}+\frac{\partial \rho_{i} v_{i z} v_{i z}}{\partial z}+(2-i) \frac{\partial p}{\partial z}=(-1)^{i}\left(F_{z}-J v_{2 z}\right) \text {, } \\
& \frac{\partial \rho_{2} e_{2}}{\partial t}+\frac{\partial \rho_{2} e_{2} v_{2 r} r}{r \partial r}+\frac{\partial \rho_{2} e_{2} v_{2 z}}{\partial z}=Q \eta(-J)-J e_{2}, \\
& \sum_{i=1}^{2}\left[\frac{\partial \rho_{i} E_{i}}{\partial t}+\frac{\partial\left(\rho_{i} E_{i}+\alpha_{i} p\right) v_{i r} r}{r \partial r}+\frac{\partial\left(\rho_{i} E_{i}+\alpha_{i} p\right) v_{i z}}{\partial z}\right]=0 \\
& \rho_{11}=\rho_{11}^{0} \alpha_{11} \rho_{12}=\rho_{12}^{0} \alpha_{11}, \quad \rho_{1}=\rho_{1}^{0} \alpha_{1}, \quad \rho_{1}^{0}=\sum_{k=1}^{2} \rho_{1 k}^{0}, \quad \rho_{1}=\rho_{11}+\rho_{12}, \quad \rho_{2}=\rho_{2}^{0} \alpha_{2} \text {, } \\
& v_{i}^{2}=v_{i r}^{2}+v_{i z}^{2}, \quad \alpha_{2}=\frac{1}{6} \pi d^{3} n, \quad \alpha_{1}+\alpha_{2}=1, \quad E_{i}=e_{i}+0.5 v_{i}^{2}, \quad(i=1,2), \quad \eta(z)= \begin{cases}0, & z<0 \\
1, & z \geq 0 .\end{cases}
\end{aligned}
$$

Здесь представлены уравнения сохранения масс инертного газа $(k=1)$, газообразных продуктов горения $(k=2)$ и частиц унитарного топлива, уравнение сохранения числа дисперсных включений, уравнения сохранения импульсов фаз в проекциях на оси декартовой системы координат $r$ и $z$, уравнение притока тепла к дисперсной фазе и уравнение сохранения полной энергии смеси. Индексы “1” и “2" внизу относятся к параметром газовой и дисперсной фаз; $\rho_{1 k}$ и $\rho_{1 k}^{0}$ - приведенная и истинная плотности $k-$ й компоненты газовой фазы; $\rho_{i}, \rho_{i}^{0}, v_{i}, \alpha_{i}, e_{i}, E_{i}$ соответственно средняя и истинная плотности, массовая скорость, объемное содержание, удельные внутренняя и полная энергии $i$-й фазы $(i=1,2) ; v_{i r}$ и $v_{i z}$ - компоненты скорости $v_{i} ; n-$ число дисперсных частиц в единице объема смеси; $d$ диаметр частиц; $p$-давление газовой смеси; $F_{r}$ и $F_{z}$ - составляющие силы межфазного трения; $Q$ - интенсивность теплообмена между газовой и дисперсной фазами; $J$ - интенсивность межфазного массообмена; $\eta$ - единичная функция Хевисайда.

Используются уравнения состояния идеального калорически совершенного газа и несжимаемых твердых частиц $[1,2]$ :

$$
\begin{gathered}
p_{1 k}=\rho_{1 k}^{0} R_{1 k} T_{1}, \quad p=\sum_{k=1}^{2} p_{1 k}=\rho_{1}^{0} R_{1} T_{1}, \quad \rho_{2}^{0}=\text { const, } e_{1 k}=c_{v 1 k}\left(T_{1}-T_{0}\right)+e_{1 k}^{0}, \\
e_{1}=\sum_{k=1}^{2} x_{1 k} e_{1 k}=c_{v 1}\left(T_{1}-T_{0}\right)+e_{1}^{0}, \quad e_{2}=c_{2}\left(T_{2}-T_{0}\right)+e_{2}^{0},
\end{gathered}
$$




\begin{tabular}{|c|c|c|c|c|c|c|}
\hline \multirow{4}{*}{ Impact Factor: } & ISRA (India) & $=3.117$ & SIS (USA) & $=0.912$ & ICV (Poland) & $=6.630$ \\
\hline & ISI (Dubai, UAE & $=0.829$ & РИНЦ (Russia) & $=0.156$ & PIF (India) & $=1.940$ \\
\hline & GIF (Australia) & $=0.564$ & ESJI (KZ) & $=8.716$ & IBI (India) & $=4.260$ \\
\hline & JIF & $=1.500$ & SJIF (Morocco) & $=5.667$ & OAJI (USA) & $=0.350$ \\
\hline
\end{tabular}

$$
\begin{gathered}
e_{2}^{0}-e_{12}^{0}=Q^{0}-\frac{p_{0}}{\rho_{10}^{0}}+\frac{p_{120}}{\rho_{120}^{0}} ; R_{1}=\sum_{k=1}^{2} x_{1 k} R_{1 k}, \quad c_{v 1}=\sum_{k=1}^{2} x_{1 k} c_{v 1 k}, \\
e_{1}^{0}=\sum_{k=1}^{2} x_{1 k} e_{1 k}^{0}, \quad x_{1 k}=\frac{\rho_{1 k}}{\rho_{1}}, \quad\left(R_{1 k}, \quad c_{v 1 k}, c_{2}, e_{1 k}^{0}, e_{2}^{0}, T_{0}=\text { const } ; k=1,2\right) .
\end{gathered}
$$

где $\quad p_{1 k}, R_{1 k}, c_{v 1 k}, e_{1 k}$ парциальное $_{1}$ давление, газовая постоянная, удельная теплоемкость при постоянном объеме, удельная внутренняя энергия $k$-го газового компонента;

$x_{1 k}$ - массовая концентрация $k-$ ой

составляющей газовой смеси;

$$
R_{1}, c_{v 1} c_{2}
$$

газовая постоянная и удельная теплоемкость при постоянном объеме газовой смеси в целом, а также удельная теплоемкость частиц; $T_{i}$ температура $i$ - й фазы; $\quad Q_{-}^{0}$ теплотворная способность унитарного топлива. Нижним индексом “ 0 " обозначены параметры фаз и компонент при температуре $T_{0}$.

Система уравнений (1) и (2) замыкается путем задания законов межфазного силового и теплового взаимодействия газовой и дисперсной фаз [1;2]:

$$
\begin{gathered}
F_{j}=\frac{1}{2} C_{d} \rho_{1}^{0}\left|v_{1}-v_{2}\right|\left(v_{1 j}-v_{2 j}\right) \frac{\pi d^{2}}{4} n ; \quad j=r, z, \\
Q=\pi d \lambda_{1} N u\left(T_{1}-T_{2}\right) n, \quad C_{d}=\frac{24}{\operatorname{Re}}+\frac{4}{\sqrt{\operatorname{Re}}}+0.4, \\
\operatorname{Re}=\frac{\rho_{1}^{0}\left|v_{1}-v_{2}\right| d}{\mu_{1}} \quad N u=2+0,6 \operatorname{Re}^{1 / 2} \operatorname{Pr}^{1 / 3}, \quad \operatorname{Pr}=\frac{c_{p 1} \mu_{1}}{\lambda_{1}} \\
\left|v_{1}-v_{2}\right|=\left[\left(v_{1 z}-v_{2 z}\right)^{2}+\left(v_{1 r}-v_{2 r}\right)^{2}\right]^{1 / 2}, \\
c_{p 1}=\sum_{k=1}^{2} x_{1 k} c_{p 1 k}, \quad \lambda_{1}=\sum_{k=1}^{2} x_{1 k} \lambda_{1 k}, \quad \mu_{1}=\sum_{k=1}^{2} x_{1 k} \mu_{1 k} .
\end{gathered}
$$

Здесь $C_{d}$ - коэффициент аэродинамического сопротивления твердой сферической частицы; $\mathrm{Re}, \mathrm{Nu}, \mathrm{Pr}$ - числа Рейнольдса, Нуссельта, Прандтля; $c_{p 1}{ }_{\text {и }} c_{p 1 k}, \mu_{1}$ и $\mu_{1 k}, \lambda_{1}$ и $\lambda_{1 k}$ удельные теплоемкости при постоянном давлении, динамические вязкости и коэффициенты теплопроводности газовой смеси и целом и ее компонентов.

Интенсивность образования газообразных продуктов горения частиц унитарного топлива задается по модели послойного горения [9; 10]:

$$
J=\pi \rho_{2}^{0} d^{2} n v_{S}\left(\frac{p}{p_{0}}\right)^{\varphi} \eta\left(T_{2}-T_{S}\right)
$$

где, $T_{S}$ - температура разложения унитарного топлива, $v_{S} u \varphi$ - константы горения.

\section{Постановка задачи.}

Рассматривается простой трубопровод, составленный из труб разного диаметра, $D_{1}$ и $D_{2}$ (см. рис. 1), заполненный однородной аэровзвесью унитарного топлива. Левый конец трубы ( $\mathrm{z}=0$ ) закрыть, а правый $(\mathrm{z}=\mathrm{L})$ открыть. $\mathrm{B}$ начальный момент времени улевого торца трубы в области 0 создается возмущение газа в виде треугольной ударной волны, воспламеняющее аэровзвесь в зоне 1 узкой части трубопровода. При условии достаточности энергии инициирующей ударной волны, требуется смоделировать взрыв аэровзвеси таким образом, чтобы в узкой части трубопровода 


\begin{tabular}{llllll} 
& ISRA (India) $=\mathbf{3 . 1 1 7}$ & SIS (USA) & $=\mathbf{0 . 9 1 2}$ & ICV (Poland) & $=\mathbf{6 . 6 3 0}$ \\
Impact Factor: & ISI (Dubai, UAE) $=\mathbf{0 . 8 2 9}$ & PUHL (Russia) $=\mathbf{0 . 1 5 6}$ & PIF (India) & $=\mathbf{1 . 9 4 0}$ \\
& GIF (Australia) $=\mathbf{0 . 5 6 4}$ & ESJI (KZ) & $=\mathbf{8 . 7 1 6}$ & IBI (India) & $=\mathbf{4 . 2 6 0}$ \\
& JIF & $\mathbf{1 . 5 0 0}$ & SJIF (Morocco) $=\mathbf{5 . 6 6 7}$ & OAJI (USA) & $\mathbf{0 . 3 5 0}$ \\
\hline
\end{tabular}

сформировалась стационарная волна гетерогенной детонации, которая в последующем должна выйти в широкую часть 2 трубопровода. Требуется изучить влияние основных определяющих параметров трубопровода и газовзвесях унитарного топлива на возможных режимов распространение волны горения в зоне влияния местного сопротивления, обусловленного внезапным расшире нием двухфазного потока.

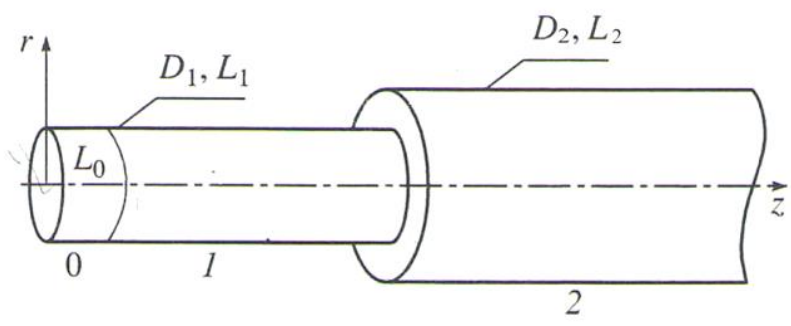

Рисунок 1 - Схематическое представление простого трубопровода: $L_{i}, D_{i}-$ длина и внутренний диаметр $i$ - го участка трубопровода $(i=1,2) ; 0$ - область ударного инициирования в газе длиной $L_{0}=z_{f}$ $L_{1}=z_{*}-L_{0} \quad L_{2}=z_{* *}-\left(L_{0}+L_{1}\right) ; z_{*}$ - осевая координата места внезапного расширения труб.

Граничные условия задачи задаются аналогично в работе [9].

Начальные условия в узкой части трубопровода принимаются аналогично

$$
\begin{gathered}
v_{1, z}=v_{1, r}=0 ; \quad p=p_{0} ; \quad T_{1}=T_{0} ; \quad \rho_{1}^{0}=\left(\rho_{1,1}^{0}\right) ; \quad \rho_{1,2}=0 ; \quad \rho_{1}=\rho_{1}^{0} \alpha_{1} ; \\
v_{2, z}=v_{2, r}=0 ; \quad T_{2}=T_{0} ; \quad \alpha_{1}=1-\alpha_{2} ; \quad \rho_{2}=\rho_{2}^{0} \alpha_{2} ; \quad \alpha_{2}=\alpha_{2,0} ; \quad n=n_{0} . \\
z_{*} \leq z \leq z_{* *} ; \quad 0 \leq r \leq R_{3} . \\
v_{1, z}=v_{1, r}=0 ; \quad p=p_{0} ; \quad T_{1}=T_{0} ; \quad \rho_{1}^{0}=\left(\rho_{1,1}^{0}\right) ; \quad \rho_{1,2}=0 ; \quad \rho_{1}=\rho_{1}^{0} \alpha_{1} ; \quad \alpha_{1}=1 . \\
z_{*} \leq z \leq z_{* *} ; \quad R_{3}<r \leq R_{2} .
\end{gathered}
$$

Задача решалась численно методом крупных частиц [11]. Расчеты выполнялись для смесей воздуха и частиц пороха.

Все вычисления осуществлялись для длины инициирующей ударной волны $z_{f}=0.4 \mathcal{M}$. В основной серии расчетов полагалось, что число Маха ударной волны $M_{0}=9$. Координаты $z_{f}, z_{*}$ и $z_{\text {** }}$ полагались равными $0.4,25$ и 50 м. В расчетах радиус узкой части трубопровода $\left(R_{1}\right)$ варьировался в интервале $0.04 \leq R_{1} \leq 0.3 \mathrm{M}$, а радиус его широкой части $\left(R_{2}\right)$ варьировался в интервале $0.08 \leq R_{2} \leq 1.5$ м.

Некоторые результаты расчетов.
Ниже проведены некоторые результаты численного исследования влияния геометрии трубопровода и размера частиц на распространение детонационных волн в газовзвесях унитарного топлива.

На рис. 2 показано влияние изменения узкой и широкой части трубопровода на распространения детонационных волн. Диаметр частиц унитарного топлива составлял $d_{2}=3$ мкм, начальное относительное массовое содержание частиц в смеси $m_{2}=1$. Радиус узкой части трубопровода равен $R_{1}=0.04 \mathrm{M}$, а радиус его широкой части $R_{2}=0.12 \mathrm{м}(\mathrm{a}), R_{1}=0.1 \mathrm{M}, R_{2}=0.3 \mathrm{м}$ (б). При $R_{1}=0.04$ м, $R_{2}=0.12$ м. переход к колебательному характеру давления наблюдается лишь в начале широкой части трубопровода (рис.2(a)), а при $R_{1}=0.1$ м, $R_{2}=0.3$ м интенсивные колебания давления наблюдается на участке $25<\mathrm{z}<35$ м (рис.2(б). Возникновение интенсивных 


\begin{tabular}{llllll} 
& ISRA (India) $=\mathbf{3 . 1 1 7}$ & SIS (USA) & $=\mathbf{0 . 9 1 2}$ & ICV (Poland) & $=\mathbf{6 . 6 3 0}$ \\
Impact Factor: & ISI (Dubai, UAE) $=\mathbf{0 . 8 2 9}$ & PUHL (Russia) $=\mathbf{0 . 1 5 6}$ & PIF (India) & $=\mathbf{1 . 9 4 0}$ \\
& GIF (Australia) $=\mathbf{0 . 5 6 4}$ & ESJI (KZ) & $=\mathbf{8 . 7 1 6}$ & IBI (India) & $=\mathbf{4 . 2 6 0}$ \\
& JIF & $\mathbf{1 . 5 0 0}$ & SJIF (Morocco) $=\mathbf{5 . 6 6 7}$ & OAJI (USA) & $\mathbf{0 . 3 5 0}$ \\
\hline
\end{tabular}

колебаний обусловлено увеличением скорости поперечной детонационный волны с изменением в определенных пределах диаметров трубопровода.
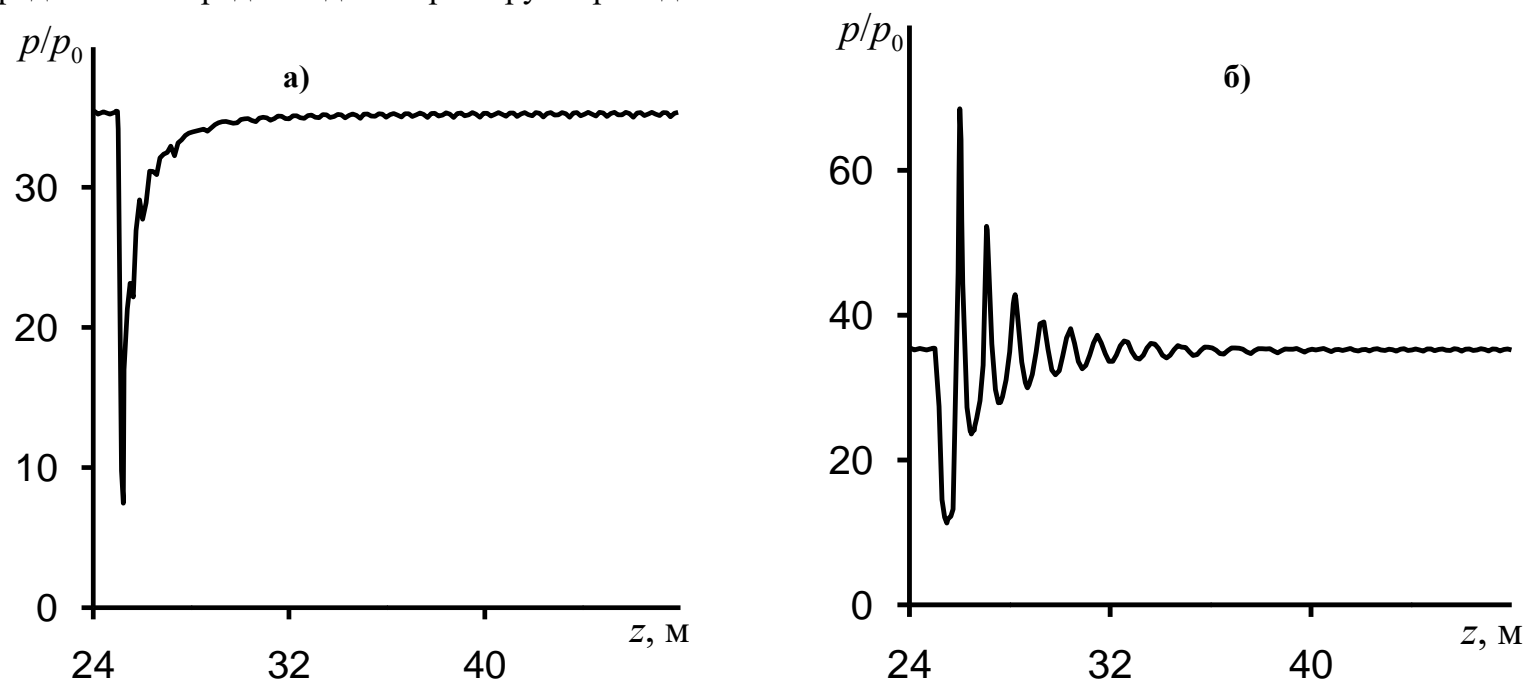

Рисунок 2 - Огибающие максимальных давлений на оси симметрии в широкой части трубопровода: $d_{2}=3$ мкм; $m_{2}=1 ; R_{1}=0.04$ м, $R_{2}=0.12$ м (a), $R_{1}=0.1$ м, $R_{2}=0.3$ м (б).

На рис. 3 показано влияние изменения широкой части трубопровода на распространения детонационных волн в газовзвесях унитарного топлива. С увеличением широкой части трубопровода интенсивность колебания давления увеличивается. В обоих случаях наблюдается продолжение детонационного процесса.
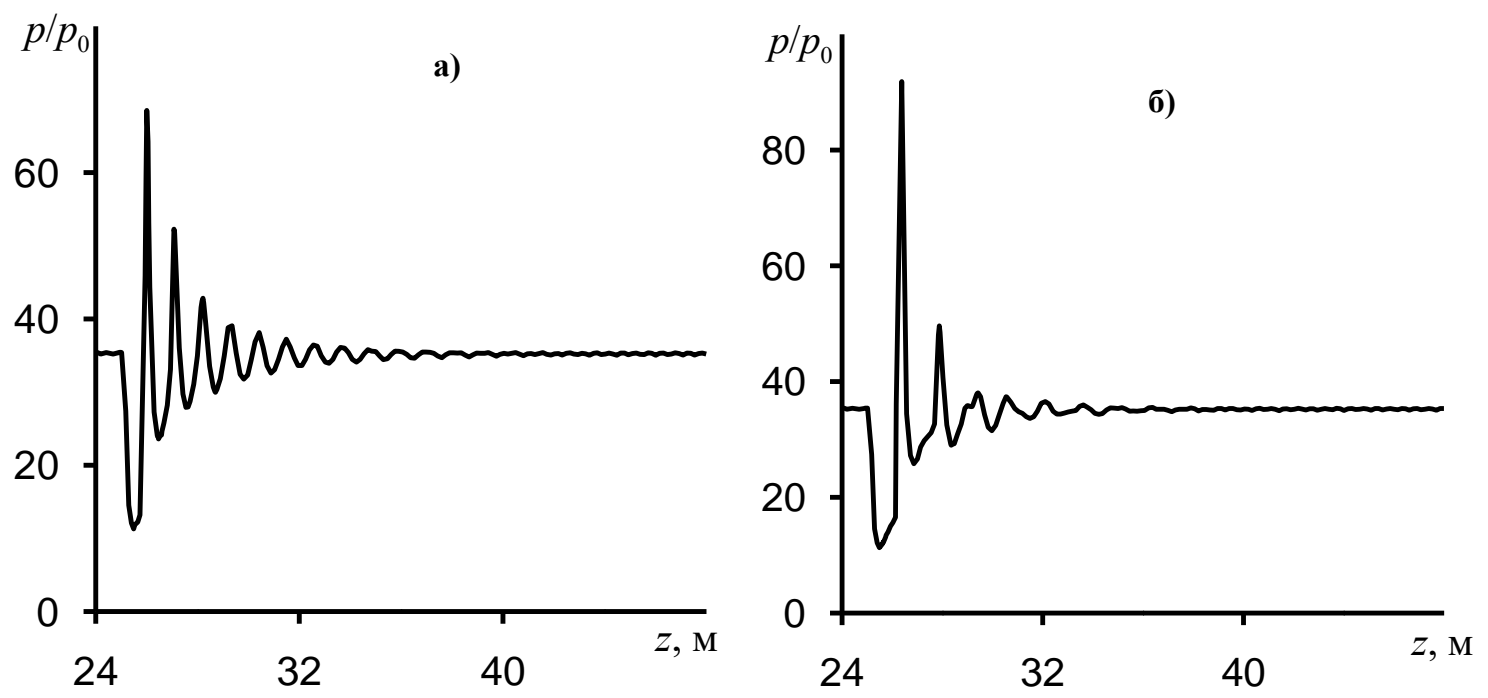

Рисунок 3 - Огибающие максимальных давлений на оси симметрии в широкой части трубопровода: $d_{2}=3$ мкм; $m_{2}=1 ; R_{1}=0.1$ м, $R_{2}=0.3$ м (а), $R_{1}=0.1$ м, $R_{2}=0.4$ м (б).

На рис. 4 приведены огибающие максимальных давлений за волнами горения на оси симметрии в широкой части трубопровода при различных диаметрах размере частиц 3 и 30 мкм. Начальное относительное массовое содержание $m_{2}=1$. Радиус узкой части трубопровода равен $R_{1}=0.2$ м, а радиус его широкой части $R_{2}=0.5$ м. Кривые 1 и 2 соответствуют частицами диаметром $d_{2}=3$ и 30 мкм. Анализ численных результатов свидетельствуют, что с увеличением размера 


\begin{tabular}{llllll} 
& ISRA (India) $=\mathbf{3 . 1 1 7}$ & SIS (USA) & $=\mathbf{0 . 9 1 2}$ & ICV (Poland) & $=\mathbf{6 . 6 3 0}$ \\
Impact Factor: & ISI (Dubai, UAE) $=\mathbf{0 . 8 2 9}$ & PUHL (Russia) $=\mathbf{0 . 1 5 6}$ & PIF (India) & $=\mathbf{1 . 9 4 0}$ \\
& GIF (Australia) $=\mathbf{0 . 5 6 4}$ & ESJI (KZ) & $=\mathbf{8 . 7 1 6}$ & IBI (India) & $=\mathbf{4 . 2 6 0}$ \\
& JIF & $\mathbf{1 . 5 0 0}$ & SJIF (Morocco) $=\mathbf{5 . 6 6 7}$ & OAJI (USA) & $\mathbf{0 . 3 5 0}$ \\
\hline
\end{tabular}

частиц восстановление детонационного процесса запаздывает.

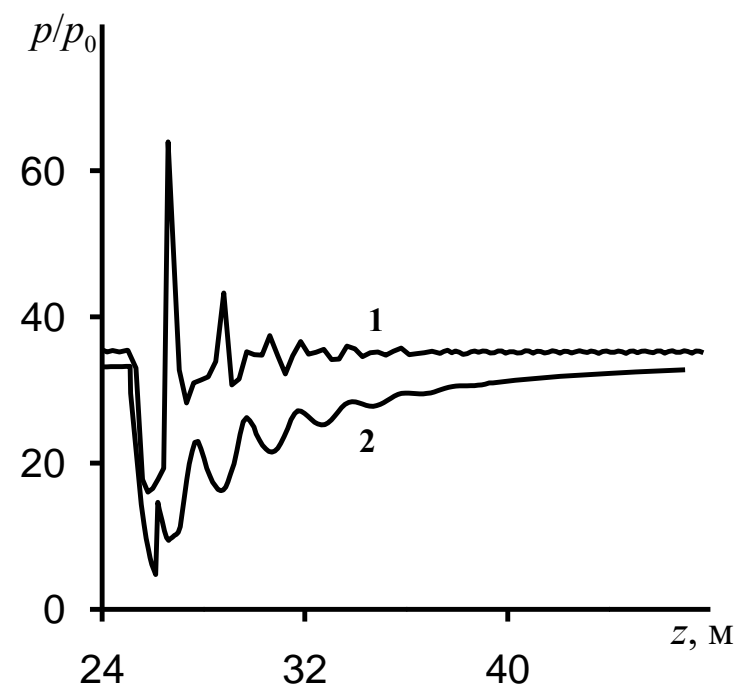

Рисунок 4 - Огибающие максимальных давлений за волнами горения частиц различного диаметра на оси симметрии в широкой части трубопровода: $m_{2}=1 ; R_{1}=0.2 \mathrm{M}, R_{2}=0.5 \mathrm{м}, 1-d_{2}=3$ мкм; $2-d_{2}=30$ мкм.

Таким образом, в результате проведенного численного исследования установлено, что размер узкой и широкой части трубопровода, а также размер частиц унитарного топлива существенно влияет на структуры детонационных волн в газовзвесях унитарного топлива.

\section{References:}

1. Nigmatulin, R. I. (1987). Dinamika mnogofaznykh sred. (p.464). Ch.1. Moscow: Nauka.

2. Kutushev, A. G. (2003). Matematicheskoe modelirovanie volnovykh protsessov $v$ aerodispersnykh i poroshkoobraznykh sredakh. (p.284). SPb: Nedra.

3. Kratova, Y. V., Fedorov, A. V., Fomin, V. M., \& Khmel', T. A. (2010). Rezhimy rasprostraneniya ploskoy detonatsii v gazovzvesyakh $\mathrm{v}$ kanalakh s razryvom secheniya. Fiziko-khimicheskaya kinetika $v$ gazovoy dinamike. Tom 9, pp.1-8. http://www.chemphys.edu.ru/media/files/201001-12-005.pdf

4. Kratova, Y. V., Fedorov, A. V., \& Khmel', T. A. (2009). Difraktsiya ploskoy detonatsionnoy volny na obratnom ustupe v gazovzvesi. $F G V, T$. 45, № 5. pp. 95-107.

5. Kratova, Y. V., Fedorov, A. V., \& Khmel', T. A. (2011). Rasprostraneniya detonatsionnykh voln $\mathrm{v}$ gazovzvesyakh $\mathrm{v}$ kanalakh $\mathrm{s}$ vnezapnym rasshireniem. Fizika goreniya i vzryva, T. 47, № 1, pp. 80-91.

6. Arienti, M., \& Shepherd, J. E. (2005). A numerical study of detonation diffraction. $J$. Fluid Mech. 529, pp. 117-146.

7. Pantov, E. G., Fischer, M., \& Kratzel, T. (1996). Decoupling and recoupling of detonation waves associated with sudden expansion. (pp. 131137). Shock Waves.

8. Fedorov, A. V., Kratova, Y. V., Khmel', T. A., \& Fomin, V. M. (2008). Rasprostranenie udarnykh i detonatsionnykh voln $\mathrm{v}$ kanalakh razlichnoy geometrii v gazovzvesyakh. Fizikokhimicheskaya kinetika $v$ gazovoy dinamike. Tom 7. Elektronnyy zhurnal, nomer gosudarstvennoy registratsii 0420700013 . ISSN 1991-6396

http://www.chemphys.edu.ru/media/files/200809-01-006.pdf

9. Kutushev, A. G., \& Shorokhova, L. V. (2003). Chislennoe issledovanie protsessov goreniya $\mathrm{i}$ 


\begin{tabular}{llllll} 
& ISRA (India) $=\mathbf{3 . 1 1 7}$ & SIS (USA) $=\mathbf{0 . 9 1 2}$ & ICV (Poland) & $\mathbf{= 6 . 6 3 0}$ \\
Impact Factor: & ISI (Dubai, UAE) $=\mathbf{0 . 8 2 9}$ & PUHЦ (Russia) $=\mathbf{0 . 1 5 6}$ & PIF (India) & $=\mathbf{1 . 9 4 0}$ \\
& GIF (Australia) $=\mathbf{0 . 5 6 4}$ & ESJI (KZ) & $\mathbf{8 . 7 1 6}$ & IBI (India) & $=\mathbf{4 . 2 6 0}$ \\
JIF & $\mathbf{1 . 5 0 0}$ & SJIF (Morocco) $=\mathbf{5 . 6 6 7}$ & OAJI (USA) & $\mathbf{0 . 3 5 0}$ \\
\hline
\end{tabular}

detonatsii aerovzvesey unitarnogo topliva $\mathrm{v}$ rezko rasshiryayushchikhsya trubakh. Khimicheskaya fizika, T. 22, № 8, pp.94-99.

10. Burnashev, V. F., Nazarov, U. A., \& Khuzhaerov, B. K. (2016). Detonatsionnye volny $\mathrm{V}$ polidispersnykh gazovzvesyakh unitarnogo topliva $\mathrm{v}$ rezko rasshiryayushchikhsya trubakh. Izvestiya $R A N$. Mekhanika zhidkosti i gaza, № 4, pp.72-79.

11. Belotserkovskiy, O. M., \& Davydov, Y. M. (1982). Metod krupnykh chastits v gazovoy dinamike. Moscow: Nauka. 